\title{
Aging in coherent noise models and natural time
}

\author{
Ugur Tirnakli* \\ Department of Physics, Faculty of Science, Ege University, 35100 Izmir, Turkey \\ Sumiyoshi Abe ${ }^{\dagger}$ \\ Institute of Physics, University of Tsukuba, Ibaraki 305-8571, Japan
}

\begin{abstract}
Event correlation between aftershocks in the coherent noise model is studied by making use of natural time, which has recently been introduced in complex time-series analysis. It is found that the aging phenomenon and the associated scaling property discovered in the observed seismic data are well reproduced by the model. It is also found that the scaling function is given by the $q$-exponential function appearing in nonextensive statistical mechanics, showing power-law decay of event correlation in natural time.

PACS Number(s): 89.75.Da, 05.65.+b, 05.90.+m, 91.30.-f
\end{abstract}

In recent years, there has been an increasing interest in extended dynamical systems exhibiting avalanches of activity, whose size distribution is scale-free. Examples of such systems are earthquakes [1], rice piles [2], extinction in biology [3], evolving complex networks [4], and so on. Up to now, there is no unique and unified theory for such systems, but one of the candidates may be the notion of self-organize criticality (SOC) [5]. A key feature common in SOC models is that the whole system is under the influence of a small driving force that acts locally. These systems evolve towards a critical stationary state having no characteristic spatio-temporal scales without fine-tuning parameters. Two celebrated examples of such extended driven dynamical systems are the Olami-Feder-Christensen model of earthquakes [6] and the Bak-Sneppen model for biological evolution [7].

On the other hand, there exists another kind of simple and robust mechanism producing scale-free behavior in the absence of criticality. An important example is the coherent noise model [8], which we shall study here. The coherent noise model has been introduced to describe large-scale events in evolution. It is based on the notion of external stress coherently imposed on all agents of the system under consideration. Since this model does not contain any direct interaction among agents, it does not exhibit dynamical criticality. Nevertheless, it yields a power-law distribution of event (i.e., avalanche) size $s$, which is defined by the number of agents that change their states at each time step. The model allows existence of aftershocks. This is a direct consequence of the fact that, in the coherent noise model, the probability of a large event to occur is increased immediately after a previous large event.

The coherent noise model is defined in a rather simple manner. Consider a system, which consists of $N$ agents. Each agent $i$ has a threshold $x_{i}$ against external stress $\eta$. The threshold levels are chosen at random due to some probability distribution $p_{t h r e s h}(x)$. The external stress is also chosen randomly due to another distribution $p_{\text {stress }}(\eta)$. An agent becomes eliminated if it is subjected to the stress $\eta$ exceeding the threshold for the agent. In practice, dynamics of the model can be summarized as the following three steps: (i) at each time step, a random stress $\eta$ is generated from $p_{\text {stress }}(\eta)$ and all the agents with $x_{i} \leq \eta$ become eliminated and are replaced by new agents with new thresholds drawn from $p_{\text {thresh }}(x)$, (ii) a small fraction $f$ of the $N$ agents should be chosen at random and given new thresholds, and then (iii) go back to (i) for the next time step. Here, (ii) corresponds to the probability for the $f$ fraction of the whole agents of undergoing spontaneous transition. This is necessary for preventing the model from grinding to a halt (see e.g. Ref. [9] for details).

The coherent noise model has been applied to the problems of earthquakes $[9,10]$. There, the GutenbergRichter law for the relation between frequency of the events and the values of the magnitude [11] and the Omori law for temporal decay pattern of aftershocks [12] are found to be realized. In particular, the Omori law, which is of relevance to our subsequent discussion, states that the rate of the number of aftershocks, $\rho$, after a mainshock at $t=0$ obeys

\footnotetext{
*e-mail: tirnakli@sci.ege.edu.tr

${ }^{\dagger}$ suabe@sf6.so-net.ne.jp
} 


$$
\rho \sim t^{-\tau}
$$

Empirically, the exponent $\tau$ takes a value between 0.6 and 1.5. This describes the slow power-law decay, and each of the relevant parts of the seismic time series is nonstationary. Such a time interval is referred to as the Omori regime.

Quite recently, the physical properties of correlation in the seismic time series have been studied in [13] based on analysis of the observed seismic data. It was shown that the aging phenomenon occurs inside the Omori regime but it disappears outside. The definite scaling property has also been identified for the event correlation function. These results suggest that nonstationarity of the Omori regime may have something to do with glassy dynamics.

A point of crucial importance regarding the aging phenomenon of earthquake aftershocks is that, unlike ordinary discussions of the aging phenomenon, the two-point correlation function is defined in the domain of "natural time" [14], not conventional time. The concept of natural time is a kind of an internal clock counting the discrete event number. It has successfully been applied to revealing physical essence of complex time series such as seismic electric signals, ionic current fluctuations in membrane channels and so on [14]. The fact that natural time is more fundamental than conventional continuous time is still empirical. In this respect, one may recall that the concept of continuum is recognized through continuous physical processes, whereas in the case of earthquakes one is concerned with a series of discrete events. However, clearly, more investigations are needed for deeper understanding of natural time.

Here, we discuss the physical properties of the Omori regime in the coherent noise model. We shall see that aging and scaling discovered in the observed seismic data are well reproduced by the model. The correlation function is found to have the form of the $q$-exponential function (see below), showing slow power-law decay. These results have also striking similarities with those recently obtained for the nonextensive Hamiltonian system of an infinite-range coupled rotors in the course of nonequilibrium relaxation dynamics [15] in conventional time, although the systems as well as the chosen random variables are completely different from each other. This, in turn, indicates that the aging/scaling phenomena may be quite universal in extended dynamical systems out of equilibria, no matter if critical or not.

We have carried out the numerical study of the aging phenomenon in the coherent noise model with the exponential distribution for the external stress

$$
p_{\text {stress }}(\eta)=a^{-1} \exp \left(-\frac{\eta}{a}\right) \quad(a>0)
$$

and the uniform distribution $p_{\text {thresh }}(x)(0 \leq x \leq 1)$ for the threshold level. The results obtained are in order.

First of all, we present in Fig. 1 a typical subinterval of the obtained time series of activity, where aftershocks are clearly identified. In Fig. 2, the rate of the probability of finding aftershocks larger than $s_{1}$ following the initial large event at $t=0$ is plotted with respect to the elapsed time $t$. The straight line represents the Omori law, which allows us to identify the Omori regime. We have ascertained that this result is insensitive to the threshold value $s_{1}$.

To investigate the property of event correlation, following the idea proposed in [13], we have employed as the basic random variable the time of the $n$th aftershock with an arbitrary avalanche size, $t_{n}$, where $n$ is aforementioned natural time in the setting of our problem. The two-point correlation function is given by

$$
C\left(n+n_{w}, n_{w}\right)=\frac{\left\langle t_{n+n_{w}} t_{n_{w}}\right\rangle-\left\langle t_{n+n_{w}}\right\rangle\left\langle t_{n_{w}}\right\rangle}{\left(\sigma_{n+n_{w}}^{2} \sigma_{n_{w}}^{2}\right)^{1 / 2}}
$$

where the average is understood as the ensemble average taken in association with a number of numerical runs, and the variances in the denominator are given by, $\sigma_{m}^{2}=\left\langle t_{m}^{2}\right\rangle-\left\langle t_{m}\right\rangle^{2}$. It is noticed that this averaging procedure is different from that in [13], in which the time average is used. Since the Omori regime is nonstationary, the correlation function depends not only on $n$ but also on $n_{w}$. In Fig. 3 , we present the plots of $C\left(n+n_{w}, n_{w}\right)$ for several values of "natural waiting time" $n_{w}$. There, the clear aging phenomenon can be appreciated. Furthermore, as shown in Fig. 4, collapse of these curves can nicely be realized, following the scaling relation

$$
C\left(n+n_{w}, n_{w}\right)=\tilde{C}\left(\frac{n}{n_{w}^{\alpha}}\right)
$$


where $\tilde{C}$ is a scaling function and $\alpha$ is numerically $\alpha \simeq 1.05$. This form of scaling is remarkably similar to the one presented in [13], where the observed seismic data are employed.

To find the form of the scaling function, we have examined the semi- $q$-log plot of the curve in Fig. 4, where the $q$-logarithmic function is defined by

$$
\ln _{q}(x)=\frac{x^{1-q}-1}{1-q} \quad(x>0),
$$

which is the inverse function of the $q$-exponential function

$$
e_{q}(x)=[1+(1-q) x]_{+}^{1 /(1-q)}
$$

with the notation $[z]_{+}=\max \{0, z\}$. (In the limit $q \rightarrow 1, \ln _{q}(x)$ and $e_{q}(x)$ converge to the ordinary logarithmic and exponential functions, respectively.) This pair of functions is known to play a central role in nonextensive statistical mechanics $[16,17]$. The straight line of the correlation function in the semi$q$-log plot shown in Fig. 5 indicates that the scaling function is given by the $q$-exponential. Therefore, event correlation decays slowly, following a power law. This reminds us of the recent discussion [15] about the nonextensive Hamiltonian system in the course of nonequilibrium relaxation, though the time variable employed there is the conventional one.

In conclusion, we have studied the physical properties of event correlation in the Omori regime of aftershocks in the coherent noise model. We have found that aging and scaling in natural time discovered in the observed seismic data can be reproduced remarkably well by this model. We have also found that the scaling function is given by the $q$-exponential function, and thus event correlation decays according to a power law.

\section{ACKNOWLEDGMENTS}

U.T. is supported by the Turkish Academy of Sciences, in the framework of the Young Scientist Award Program (UT/TUBA-GEBIP/2001-2-20). S. A. thanks Dr. H. Tanaka for discussions about natural time. He also thanks the financial support of the Grant-in-Aid for Scientific Research of Japan Society for the Promotion of Science. 
[1] J.F. Pacheco, C.H. Scholz and L.R. Sykes, Nature 255, 71 (1997).

[2] V. Frette et al., Nature 379, 49 (1996).

[3] R.V. Sole and J. Bascompte, Proc. Roy. Soc. London B 263, 161 (1997).

[4] R. Albert and A.L. Barabasi, Rev. Mod. Phys. 74, 47 (2002).

[5] P. Bak, C. Tang and K. Wiesenfeld, Phys. Rev. Lett. 59, 381 (1987).

[6] Z. Olami, H.J.S. Feder and K. Christensen, Phys. Rev. Lett. 68, 1244 (1992).

[7] P. Bak and K. Sneppen, Phys. Rev. Lett. 71, 4083 (1993).

[8] M.E.J. Newman, Proc. R. Soc. London B 263, 1605(1996); M.E.J. Newman and K. Sneppen, Phys. Rev. E $\mathbf{5 4}, 6226$ (1996).

[9] C. Wilke, S. Altmeyer, and T. Martinetz, Physica D 120, 401 (1998).

[10] K. Sneppen and M.E.J. Newman, Physica D 110, 209 (1997).

[11] B. Gutenberg and C. F. Richter, Seismicity of the Earth (Princeton University Press, Princeton, 1954).

[12] F. Omori, J. College of Sci. Imperial Univ. Tokyo 7, 111 (1894).

[13] S. Abe and N. Suzuki, Physica A 332, 533 (2004).

[14] P. A. Varotsos, N. V. Sarlis, and E. S. Skordas, Phys. Rev. E 66, 011902 (2002); 67, 021109 (2003); 68, 031106 (2003).

[15] M. A. Montemurro, F. A. Tamarit, and C. Anteneodo, Phys. Rev. E 67, 031106 (2003); A. Pluchino, V. Latora and A. Rapisarda, Continuum Mech. Thermodyn. 16, 245 (2004).

[16] C. Tsallis, J. Stat. Phys. 52, 479 (1988).

[17] Nonextensive Statistical Mechanics and Its Applications, edited by S. Abe and Y. Okamoto (Springer-Verlag, Heidelberg, 2001).

\section{Figure Captions}

Figure 1: A section of the time series of activity in the dimensionless units. 5000 time steps are shown after the largest shock out of the time series of 30000000 events. The aftershocks following the mainshock at $t=0$ are clearly recognized.

Figure 2: A histogram of the time distribution (i.e., the rate) of 90000 aftershocks larger than $s_{1}=10$ following the mainshock at $t=0$. The strength of the external stress in eq.(2) is $a=0.001$, and the fraction $f=5 \times 10^{-6}$ of $N=200000$ agents is chosen at random. It obeys a power law in eq.(1) with $\tau \simeq 1$. All quantities are dimensionless.

Figure 3: Dependence of the event correlation function $C\left(n+n_{w}, n_{w}\right)$ of the aftershocks larger than $s_{1}=1$ on natural time. The strength of the external stress in eq.(2) is $a=0.2$ and the fraction $f=0.01$ of $N=10000$ agents is chosen at random. The ensemble average over 120000 realizations is performed. The values of natural waiting time are $n_{w}=250,500,1000,2000$ and 5000 from bottom to top. All quantities are dimensionless.

Figure 4: Data collapse for the correlation function $C\left(n+n_{w}, n_{w}\right)$ shown in Fig. 3. The gray solid line corresponds to $e_{q}\left(-0.7 n / n_{w}^{1.05}\right)$ with $q \simeq 2.98$.

Figure 5: The semi- $q$ - $\log$ plot of the collapsed curve with $n_{w}=1000,2000$ and 5000 in Fig. 4 . The straight line shows that the scaling function is of the $q$-exponential form with $q \simeq 2.98$. 


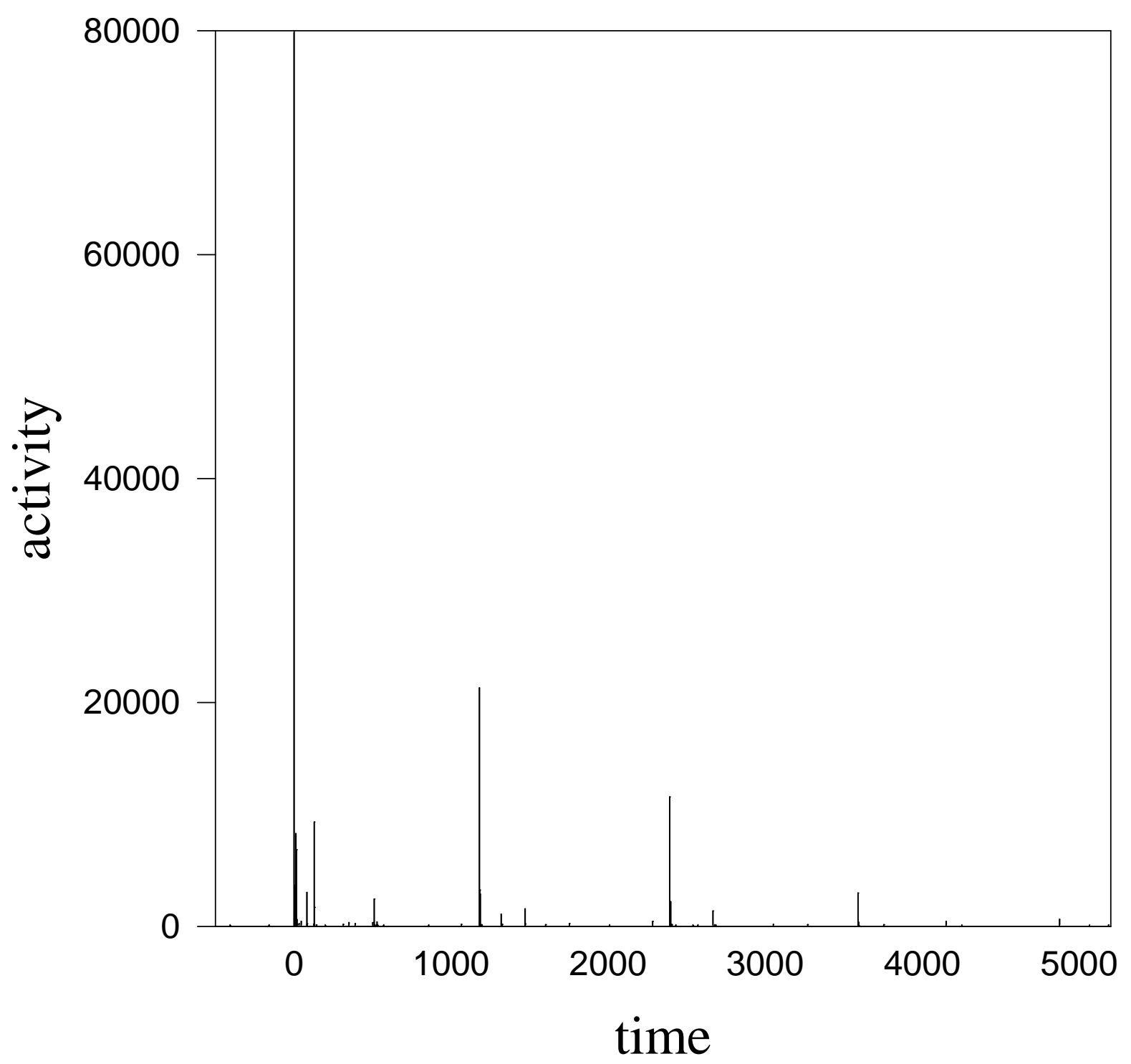




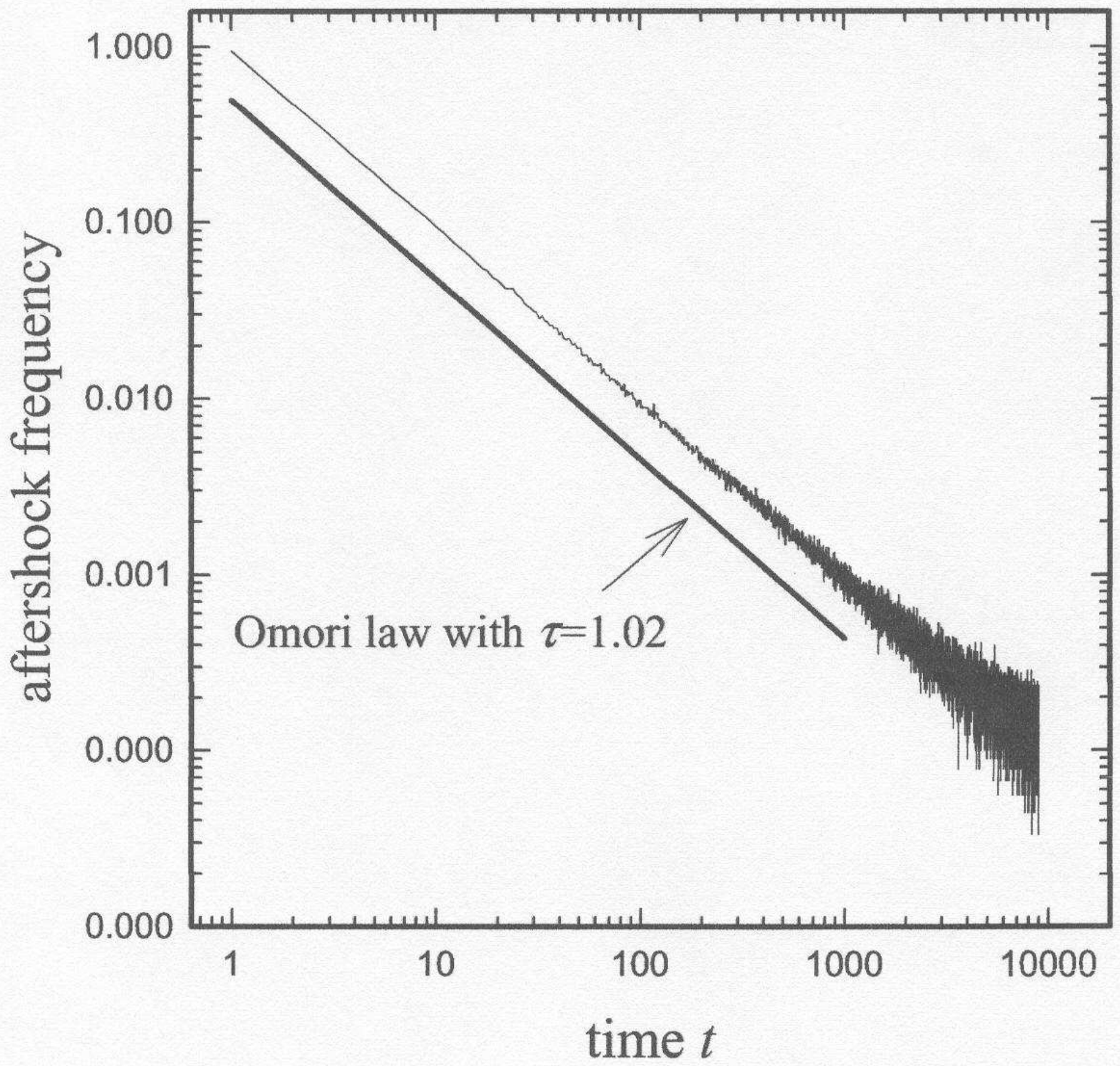




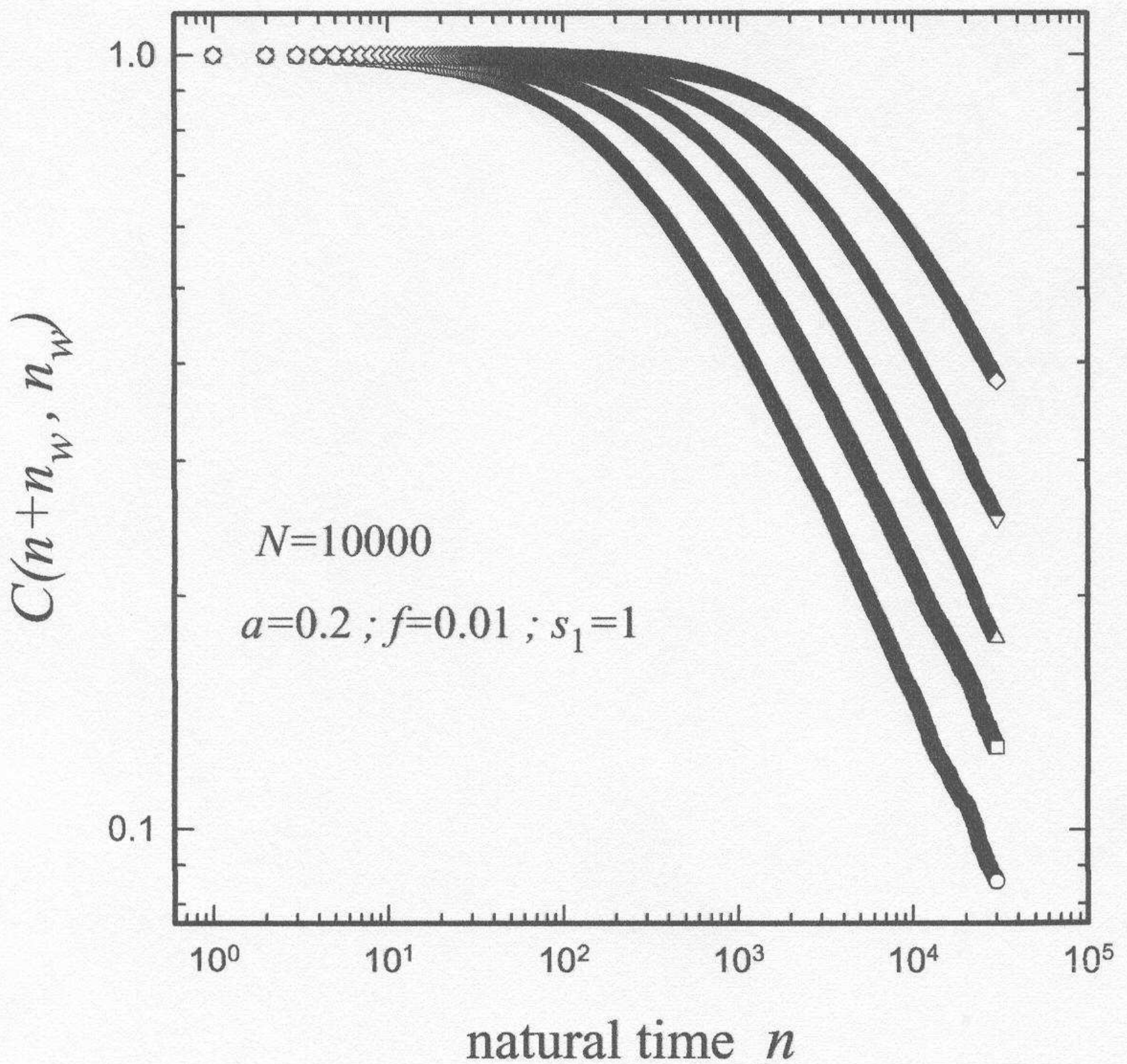




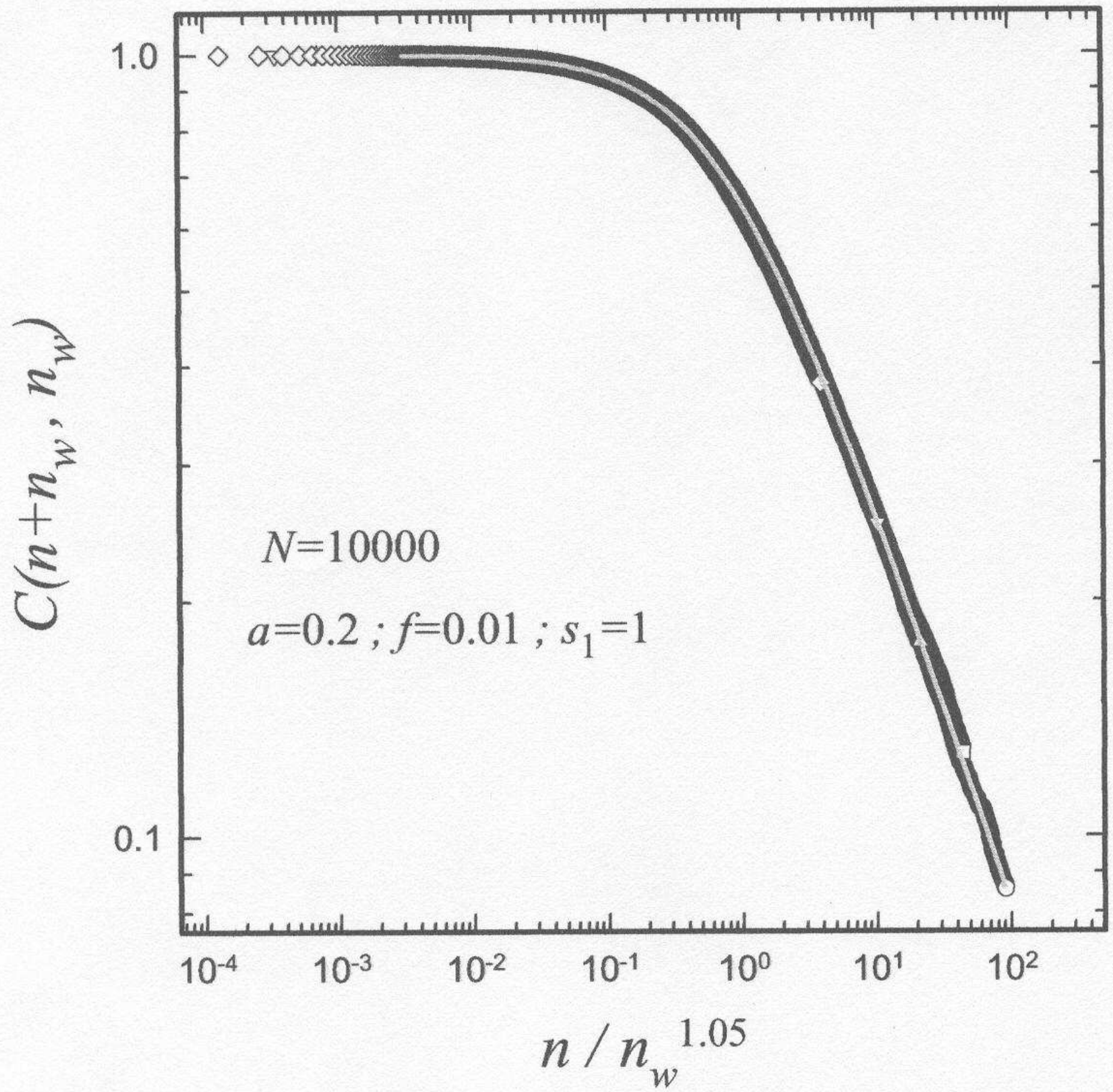




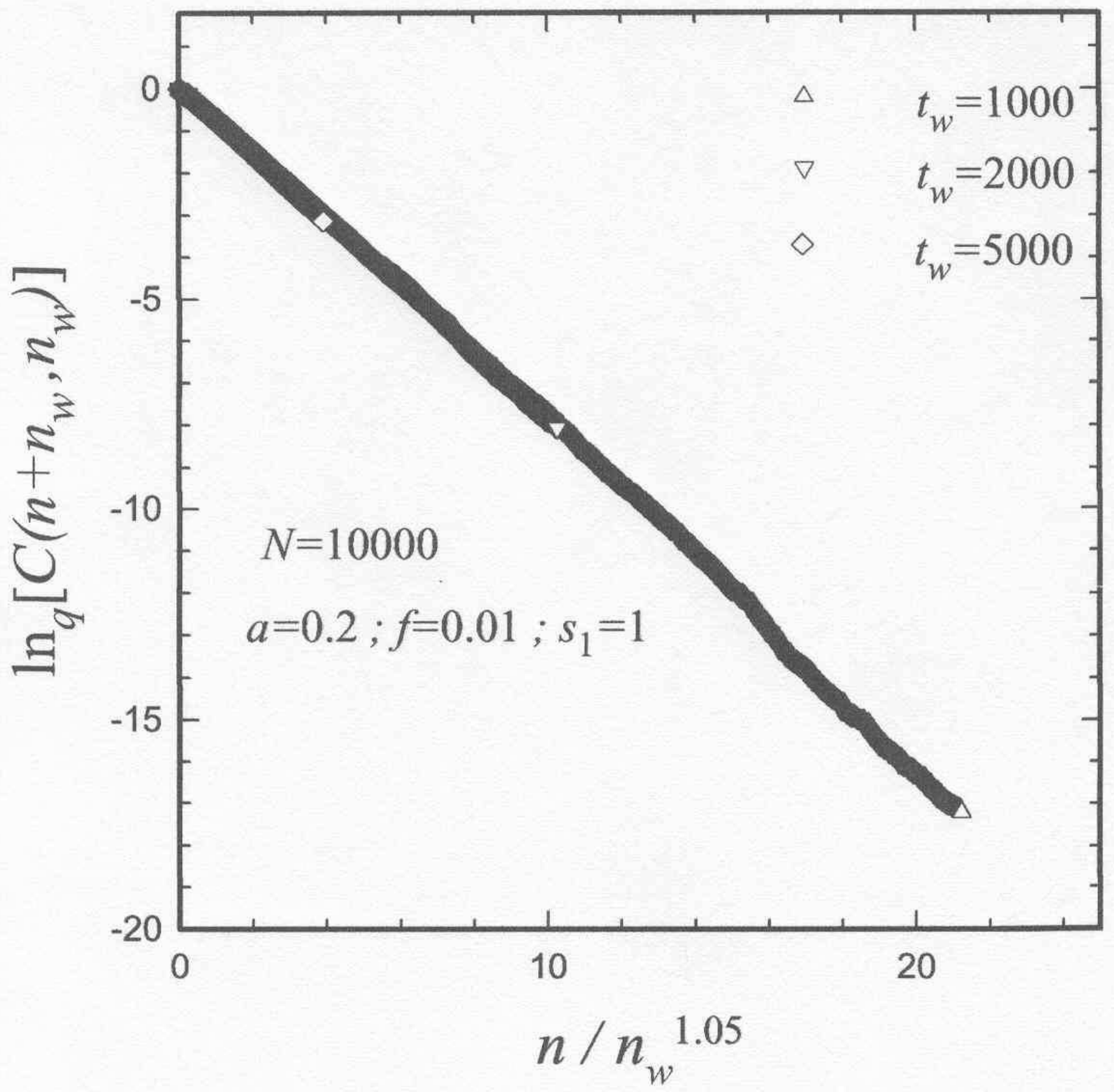

